\title{
Biofeedback Therapy Before Ileostomy Closure in Patients Undergoing Sphincter-Saving Surgery for Rectal Cancer: A Pilot Study
}

\author{
Jeong-Ki Kim ${ }^{1}$ Byeong Geon Jeon², Yoon Suk Song ${ }^{1}$, Mi Sun Seo ${ }^{1}$, Yoon-Hye Kwon ${ }^{1}$, JI Won Park ${ }^{1,2,3}$, \\ Seung-Bum Ryoo ${ }^{1}$, Seung-Yong Jeong ${ }^{1,3,4}$, Kyu Joo Park ${ }^{1}$ \\ ${ }^{1}$ Department of Surgery, Seoul National University College of Medicine, Seoul; ${ }^{2}$ Daejin Medical Center Bundang Jesaeng General Hospital, \\ Seongnam; ${ }^{3}$ Cancer Research Institute, Seoul National University, Seoul; ${ }^{4}$ Colorectal Cancer Center, Seoul National University Cancer \\ Hospital, Seoul, Korea
}

Purpose: This study prospectively investigated the effects of biofeedback therapy on objective anorectal function and subjective bowel function in patients after sphincter-saving surgery for rectal cancer.

Methods: Sixteen patients who underwent an ileostomy were randomized into two groups, one receiving conservative management with the Kegel maneuver and the other receiving active biofeedback before ileostomy closure. Among them, 12 patients (mean age, 57.5 years; range, 38 to 69 years; 6 patients in each group) completed the study. Conservative management included lifestyle modifications, Kegel exercises, and medication. Patients were evaluated at baseline and at 1, 3, 6, and 12 months after ileostomy closure by using anal manometry, modified Wexner Incontinence Scores (WISs), and fecal incontinence quality of life (FI-QoL) scores.

Results: Before the ileostomy closure, the groups did not differ in baseline clinical characteristics or resting manometric parameters. After 12 months of follow-up, the biofeedback group demonstrated a statistically significant improvement in the mean maximum squeezing pressure (from 146.3 to $178.9, \mathrm{P}=0.002$ ). However, no beneficial effect on the WIS was noted for biofeedback compared to conservative management alone. Overall, the FI-QoL scores were increased significantly in both groups after ileostomy closure $(\mathrm{P}=0.006)$, but did not differ significantly between the two groups.

Conclusion: Although the biofeedback therapy group demonstrated a statistically significant improvement in the maximum squeezing pressure, significant improvements in the WISs and the FI-QoL scores over time were noted in both groups. The study was terminated early because no therapeutic benefit of biofeedback had been demonstrated.

Keywords: Feedback; Ileostomy; Manometry; Fecal incontinence

\section{INTRODUCTION}

An ileostomy is often performed when the probability of inflammation or leakage at an anastomotic site after surgery for inflam-

Received: June 8,2015 - Accepted: July 8, 2015

Correspondence to: Kyu Joo Park, M.D.

Division of Colorectal Surgery, Department of Surgery, Seoul National University College of Medicine, Seoul National University Hospital, 101 Daehak-ro, Jongno-gu, Seoul 03080, Korea

Tel: +82-2-2072-2901, Fax: +82-2-766-3975

E-mail:kjparkmd@plaza.snu.ac.kr

(c) 2015 The Korean Society of Coloproctology

This is an open-access article distributed under the terms of the Creative Commons Attribution NonCommercial License (http://creativecommons.org/licenses/by-nc/3.0) which permits unrestricted noncommercial use, distribution, and reproduction in any medium, provided the original work is properly cited. matory or ischemic bowel diseases or surgery for colorectal cancer is high. The procedure prevents feces from reaching the anastomotic site by the creation of a stoma through which the ileum is projected outside the abdominal wall, immobilized, and resected. An ileostomy in most cases is performed as a temporary measure, so ileostomy closure is performed to reinsert the ileostomy into the abdomen 3-6 months later once the anastomosis is completed $[1,2]$.

Conservative management, such as diet control and self-anal sphincter exercise, are performed because both diarrhea and an urgent defecation desire are present for a certain time after ileostomy closure. These symptoms are thought to result from diversion colitis or anorectal function deterioration because the colon is not used during an ileostomy [3]. Although most of these 
symptoms improve over time, patients experience significant symptom-induced discomfort and need to wear a diaper or pad, which makes the fecal-incontinence quality of life (FI-QoL) worse.

Biofeedback is a self-control technique for patients who have diarrhea or fecal incontinence. Patients practice to control the movements of the external anal sphincter muscles (Kegel maneuver) and the pelvic floor muscles. The effect of biofeedback is still controversial; however, a number of studies have reported reduced incidence of diarrhea and reduced urgent defecation desire [4-7]. Especially for fecal incontinence, not only sphincter muscle exercises but also rectal sensory training is used to control the urgent desire to defecate. However, little is known about the change in and the treatment of the defecation pattern after ileostomy closure for patients who undergo sphincter-saving surgery. Therefore, we investigated the effects of anorectal biofeedback on patients' defecation patterns and FI-QoL after ileostomy closure.

\section{METHODS}

Sixteen patients who underwent ileostomy closure at the Department of Surgery, Seoul National University Hospital, from July 2009 to February 2011 voluntarily participated in this study. After an appropriate recovery period after adjuvant chemoradiotherapy, patients underwent ileostomy closure after the stable status of patients had been confirmed at the outpatient clinic. No patients had any signs of anal disease or neurological disease. Patients with uncontrolled internal medicine diseases, such as hypertension and diabetes, were excluded from the study. From the original $16 \mathrm{pa}-$ tients, 12 (8 men, 4 women) completed the study. The random placement table was managed in a blinded manner by the surgery department's outpatient clinic. When ileostomy closure was chosen for a patient after ileostomy follow-up, a third-party outpatient nurse not involved in this study disclosed the corresponding order (sequence) of the random placement table. Random placement was conducted by the Medical Research Collaboration Center (MRCC, 2009-0168) before the start of the clinical study and after the Institutional Review Board's review. Each patient was fully briefed on the background and the aim of this study, the effects and the limitations of conservative management and biofeedback, and the complications that might develop during the clinical trial. Each patient received assurances that their information and information would be held in strict confidence and was a volunteer to participate in the study.

Patients in the conservative group (CG) received training on defecation habits, the Kegel maneuver, and diet control prior to ileostomy closure, and conservative management such as pharmacological treatment was provided during outpatient follow-up. The patients of the biofeedback group (BG) received biofeedback before surgery, along with conservative management as well as intensive individual defecation education at each biofeedback session.

Defecation habit education was provided to ensure that normal defecation effectively occurred at the same time each day after a meal and in the correct position. The Kegel maneuver is a movement in which the anal external sphincter muscle is contracted by squeezing with the maximal possible force and is then relaxed. Patients were trained to perform a set number of exercises each day to strengthen the sphincter muscle and were advised to control their intakes of water and fiber in the form of vegetables and fruits and to limit their consumption of caffeine and alcohol.

Three types of biofeedback therapy methods were used: visual, auditory, and verbal. Patients were trained while watching a monitor and practiced contracting and relaxing the sphincter muscle twice a week before surgery. After surgery, intensive training was conducted for 4 weeks. In the beginning, patients experienced 'trail and error' with respect to voluntary muscles that they could not yet control; in this manner, their ability to control those muscles gradually increased. Exercises to strengthen the pelvic muscles without using the adjacent abdominal, thigh, and hip muscles were practiced, and contraction exercises began only after the contraction of a pelvic-floor-muscle had been confirmed. Patients were then instructed to breathe naturally without stopping during the pelvic muscle contraction exercise; then, they were asked to slowly contract and hold the muscle tightly, after which they were allowed a break. The strength and the number of the exercises were gradually increased, at which time patients compared their feeling of muscle control with the graphical change in muscle control shown on a monitor. After repeated practice, they were able to practice to a certain extent without verification on the monitor.

A therapist gave feedback on whether a patient was using additional muscles or breathing well and gave encouragement to motivate the patients not to succumb to fatigue and frustration when the therapy did not visibly alleviate their symptoms. Manometry and questionnaire surveys about the defecation pattern and the Fi-QoL were conducted before surgery and during outpatient postoperative visits at 1, 3, 6, and 12 months. Manometry was conducted by using the Dynacompact (Menfis Biomedica Corp., Bologna, Italy) unit located in the anorectal laboratory in the outpatient clinic, Department of Surgery, Seoul National University Hospital, to measure the anorectal pressure. A convenient suppository was used to empty the rectum before the manometry, and abnormal findings such as pain or bleeding in the anus were checked by using a digital rectal examination. The rapid pullthrough method passes the pressure-measuring catheter through the anal sphincter muscle at a constant speed and measures the anal sphincter's length, high pressure zone, vector volume, and sphincter asymmetry. The stationary pull-through method measures the resting and the squeezing pressures at the stationary state, along with the rectoanal inhibitory reflex, the sensory threshold, the rectal capacity and compliance, and the bear down. The fecal incontinence level in each patient was measured by using the Wexner Incontinence Score (WIS). The defecation pattern was surveyed through a questionnaire by using the Bristol stool scale while the FI-QoL was surveyed by using a questionnaire. 
The WIS is a fecal incontinence index that scores fecal incontinence types (solid, liquid, and gas), diaper use, and lifestyle changes according to incontinence frequency as follows: 4 , more than once a day; 3 , once a day or week; 2 , less than once a week or month; 1 , less than once a month; and 0 , no incontinence. A score of 20 points indicated complete fecal incontinence [8]. The FIQoL questionnaire was composed of 29 items: 10 on lifestyle, 9 on coping and behavior, 7 on depression and self-perception, and 3 on embarrassment [9]. Each item received a score from 1 to 4 points, and a lower total score indicates a lower FI-QoL.

Statistical analysis was performed by using SPSS ver. 17.0 (SPSS Inc., Chicago, IL, USA). Categorical variables were compared between the two groups by using Pearson chi-square test or Fisher exact test. Continuous variables were compared between the two groups by using Student $\mathrm{t}$-test. A P-value $<0.05$ was considered significant.

\section{RESULTS}

Prior to ileostomy closure, no differences between the BG and the CG were noted with respect to age, sex, preoperative chemoradiation therapy before surgery, surgery method, distance to the tumor, tumor stage, duration until ileostomy closure, and rectal resting pressure (Table 1). Twelve months after ileostomy closure, the maximum squeezing pressure had increased from 146.3 (baseline) to $178.9(\mathrm{P}=0.002)$ in the $\mathrm{BG}$, but had remained relatively the same in the CG $(P=0.838)$. No statistical differences in the other rectal pressure measurements (resting pressure, sustained duration, sphincter length, and high pressure zone) were found between the BG and the CG during the follow-up period (Table 2, Fig. 1).

The mean WISs during the follow-up period, measured at 1, 3, 6, and 12 months, after ileostomy closure decreased from 9.0 to 6.2 in the $\mathrm{BG}$ and from 10.7 to 7.0 in the $\mathrm{CG}(\mathrm{P}=0.031)$. However, no therapeutic benefit of biofeedback therapy was seen on the in-

Table 1. Demographic and baseline clinical characteristics of the biofeedback (BG) and the conservative groups (CG)

\begin{tabular}{lccc}
\hline Characteristic & BG $(\mathrm{n}=6)$ & $\mathrm{CG}(\mathrm{n}=6)$ & P-value \\
\hline Age (yr) & $60.6 \pm 6.0$ & $54.5 \pm 10.1$ & 0.229 \\
Sex & & & \\
$\quad$ Male:female & $3: 3$ & $5: 1$ & 0.545 \\
Preoperative CCRT & $5(83.3)$ & $5(83.3)$ & 1.000 \\
Primary operation & & & 1.000 \\
LAR (end-to-end anastomosis) & $3(50)$ & $4(66.7)$ & \\
ULAR (end-to-end anastomosis) & $3(50)$ & $2(33.3)$ & \\
Tumor location from AV (cm) & $4.5 \pm 1.5$ & $5.5 \pm 5.0$ & 0.936 \\
Time duration from ileostomy & $274.3 \pm 93.2$ & $310.2 \pm 113.7$ & 0.749 \\
to closure (day) & & & \\
Tumor stage & & & 0.849 \\
I & 1 & 2 & \\
II & 3 & 1 & \\
III & 2 & 4 & \\
IV & 1 & 0 & \\
Manometric parameters & & & \\
RASP (mmHg) & $44.7 \pm 11.3$ & $44.4 \pm 18.8$ & 1.000 \\
MASP (mmHg) & $146.3 \pm 25.6$ & $142.5 \pm 55.5$ & 0.874 \\
Sustained duration (sec) & $3.2 \pm 1.2$ & $3.5 \pm 1.7$ & 0.688 \\
Sphincter length (cm) & $4.0 \pm 1.0$ & $4.1 \pm 1.0$ & 0.810 \\
High pressure zone (cm) & $2.7 \pm 1.4$ & $2.2 \pm 0.6$ & 0.516 \\
\hline
\end{tabular}

Values are presented as mean \pm standard deviation or number (\%).

CCRT, concurrent chemoradiation therapy; LAR, low anterior resection; ULAR, ultra LAR; AV, anal verge; RASP, resting anal squeezing pressure; MASP, maximum anal squeezing pressure.

Table 2. Anorectal physiological measurements for the BG and the CG

\begin{tabular}{|c|c|c|c|c|c|c|c|c|}
\hline \multirow{2}{*}{$\begin{array}{l}\text { Anorectal physiological } \\
\text { measurement }\end{array}$} & \multirow{2}{*}{ Group } & \multirow{2}{*}{ Preoperation } & \multirow{2}{*}{1 Month } & \multirow{2}{*}{3 Months } & \multirow{2}{*}{6 Months } & \multirow{2}{*}{12 Months } & \multicolumn{2}{|c|}{$P$-value } \\
\hline & & & & & & & Within group & Between groups \\
\hline \multirow[t]{2}{*}{$\mathrm{RASP}(\mathrm{mmHg})$} & $B G$ & $44.7 \pm 11.3$ & $40.9 \pm 12.4$ & $33.6 \pm 18.2$ & $39.1 \pm 10.7$ & $41.1 \pm 12.6$ & 0.094 & 0.899 \\
\hline & $C G$ & $44.1 \pm 25.5$ & $34.5 \pm 21.8$ & $36.0 \pm 20.5$ & $42.0 \pm 29.3$ & $45.9 \pm 30.4$ & & \\
\hline \multirow[t]{2}{*}{ MASP (mmHg) } & $B G$ & $146.3 \pm 25.6$ & $161.8 \pm 46.6$ & $175.1 \pm 44.8$ & $181.5 \pm 44.9$ & $178.9 \pm 38.4$ & 0.002 & 0.838 \\
\hline & $C G$ & $142.5 \pm 55.5$ & $159.6 \pm 47.2$ & $161.6 \pm 44.9$ & $175.5 \pm 58.3$ & $176.0 \pm 78.7$ & & \\
\hline \multirow[t]{2}{*}{ Sustained duration (sec) } & $B G$ & $3.2 \pm 1.2$ & $2.7 \pm 0.8$ & $4.9 \pm 2.3$ & $5.0 \pm 2.5$ & $3.5 \pm 1.0$ & 0.139 & 0.585 \\
\hline & $C G$ & $3.5 \pm 1.7$ & $4.8 \pm 2.9$ & $4.1 \pm 1.6$ & $3.3 \pm 0.8$ & $2.3 \pm 0.7$ & & \\
\hline \multirow[t]{2}{*}{ Sphincter length (cm) } & $B G$ & $4.0 \pm 1.0$ & $4.1 \pm 0.7$ & $4.0 \pm 0.8$ & $3.6 \pm 0.7$ & $3.7 \pm 0.7$ & 0.246 & 0.717 \\
\hline & $C G$ & $4.1 \pm 1.0$ & $4.3 \pm 0.5$ & $4.0 \pm 1.2$ & $4.1 \pm 0.9$ & $3.6 \pm 0.9$ & & \\
\hline \multirow[t]{2}{*}{ High pressure zone (cm) } & $B G$ & $2.7 \pm 1.4$ & $2.5 \pm 0.7$ & $2.4 \pm 0.4$ & $2.4 \pm 0.6$ & $2.1 \pm 0.2$ & 0.970 & 0.803 \\
\hline & $\mathrm{CG}$ & $2.2 \pm 0.6$ & $2.6 \pm 0.5$ & $2.5 \pm 0.4$ & $2.6 \pm 1.1$ & $2.6 \pm 1.1$ & & \\
\hline
\end{tabular}

Values are presented as mean \pm standard deviation.

$\mathrm{BG}$, biofeedback group; CG, conservative group; RASP, resting anal squeezing pressure; MASP, maximum anal squeezing pressure. 
tergroup comparison $(\mathrm{P}=0.219)$. While the FI-QoL significantly increased in both groups after ileostomy closure, no significant difference was observed between the two groups $(\mathrm{P}<0.001$ vs. $\mathrm{P}$ $=0.976)$. In particular, lifestyle and coping in the FI-QoL were significantly improved in both groups after surgery $(\mathrm{P}<0.001, \mathrm{P}$ $<0.001$, respectively) whereas depression and embarrassment showed no statistically significant differences between the two groups ( $\mathrm{P}=0.398, \mathrm{P}=0.163$, respectively) (Table 3, Fig. 2).

\section{DISCUSSION}

After an ileostomy, patients experience discomfort due to diarrhea and an urgent desire to defecate. The present study is a prospective, randomized study based on questionnaire surveys. We investigated not only objective values such as the effects of biofeedback therapy or conservative therapy and measurements of the rectal pressure but also subjective values such as the WISs and
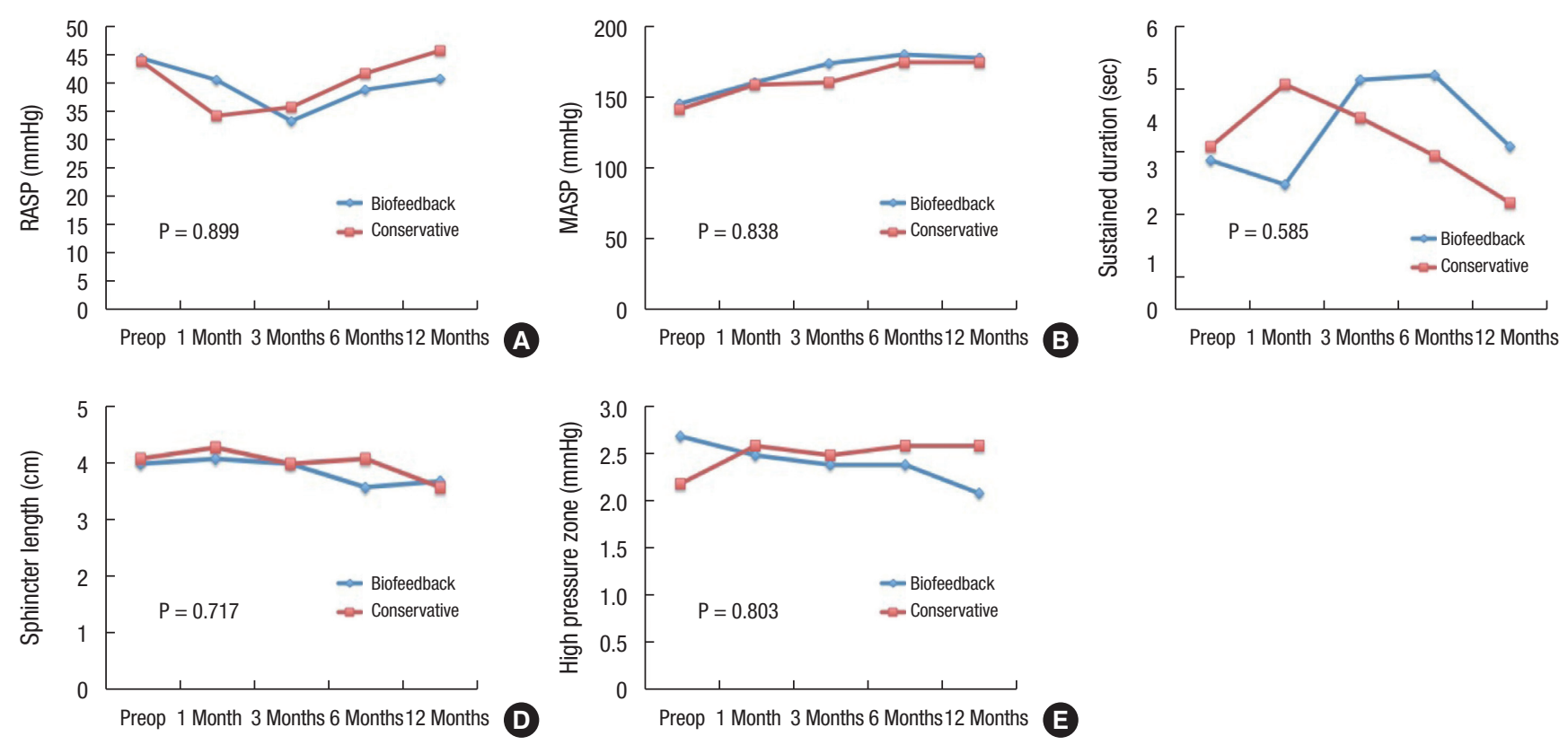

Fig. 1. Anorectal physiological measurements for the biofeedback group and the conservative group: (A) resting pressure, (B) squeezing pressure, (C) sustained duration, (D) sphincter length, and (E) high pressure zone. RASP, resting anal squeezing pressure; MASP, maximum anal squeezing pressure; Preop, preoperation.

Table 3. Incontinence and quality of life for the BG and the CG

\begin{tabular}{|c|c|c|c|c|c|c|c|}
\hline \multirow{2}{*}{ Incontinence and QoL } & \multirow{2}{*}{ Group } & \multirow{2}{*}{1 Month } & \multirow{2}{*}{3 Months } & \multirow{2}{*}{6 Months } & \multirow{2}{*}{12 Months } & \multicolumn{2}{|c|}{ P-value } \\
\hline & & & & & & Within group & Between groups \\
\hline Wexner Incontinence Score & $B G$ & $9.0 \pm 3.5$ & $10.5 \pm 2.7$ & $8.3 \pm 2.7$ & $6.2 \pm 1.7$ & 0.031 & 0.219 \\
\hline \multicolumn{8}{|l|}{ FI-QoL subscales } \\
\hline Life style & $B G$ & $22.2 \pm 10.4$ & $25.0 \pm 9.8$ & $28.7 \pm 7.3$ & $32.8 \pm 4.7$ & $<0.001$ & 0.976 \\
\hline \multirow[t]{2}{*}{ Coping/behavior } & $B G$ & $20.0 \pm 7.3$ & $22.2 \pm 6.2$ & $24.5 \pm 4.8$ & $26.5 \pm 5.4$ & $<0.001$ & 0.979 \\
\hline & CG & $17.3 \pm 8.0$ & $21.8 \pm 7.0$ & $28.3 \pm 4.5$ & $26.0 \pm 8.0$ & & \\
\hline \multirow[t]{2}{*}{ Depression/self perception } & $B G$ & $17.8 \pm 6.2$ & $14.7 \pm 5.5$ & $17.0 \pm 3.6$ & $16.7 \pm 4.2$ & 0.398 & 0.948 \\
\hline & $C G$ & $14.3 \pm 5.4$ & $17.2 \pm 2.8$ & $19.0 \pm 2.0$ & $16.2 \pm 3.2$ & & \\
\hline Embarrassment & $B G$ & $8.5 \pm 3.2$ & $9.5 \pm 1.9$ & $10.5 \pm 1.5$ & $10.2 \pm 1.7$ & 0.163 & 0.840 \\
\hline
\end{tabular}

Values are presented as mean \pm standard deviation.

$\mathrm{BG}$, biofeedback group; CG, conservative group; FI-QoL, fecal incontinence quality of life. 

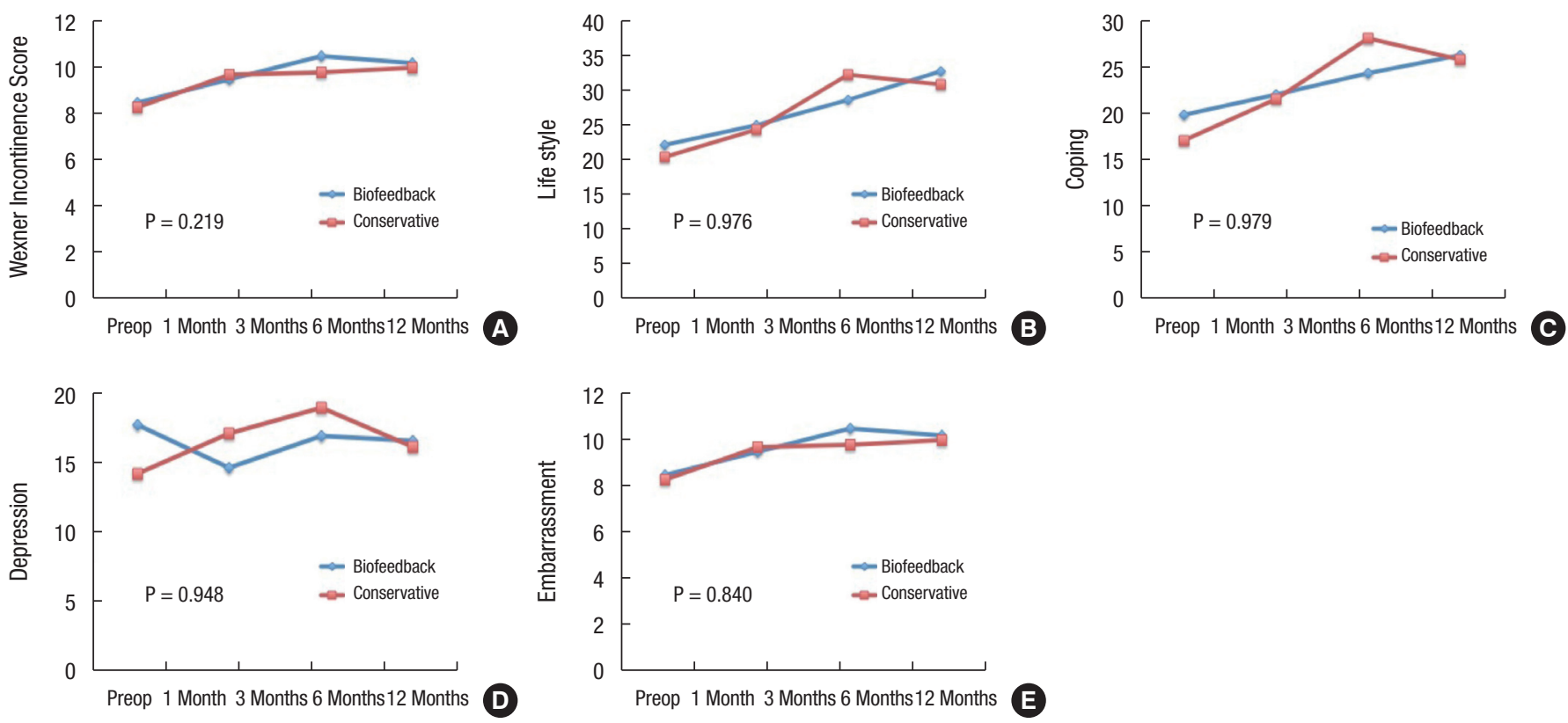

Fig. 2. Fecal incontinence quality of life for the biofeedback group and the conservative group: (A) Wexner Incontinence Score, (B) life style, (C) coping/behavior, (D) depression/self perception, and (E) embarrassment.

the FI-QoL scores. Even though the number of participating patients was small and biofeedback therapy was halted during the study due to the lack of a specific benefit, we found that the WIS was decreased in both the BG and the CG. Furthermore, we also found that lifestyle and coping factors for the FI-QoL were improved. These results suggest that both biofeedback therapy and conservative therapy are beneficial for treating fecal incontinence.

Anterior resection syndrome is a phenomenon that results from sphincter-saving surgery used to treat rectal cancer and includes a variety of symptoms such as fecal incontinence and constipation [10]. Anterior resection syndrome is considered a temporary symptoms and is usually improved a year after surgery [11]. However, Bryant et al. [12] reported that the symptoms could last up to 15 months after surgery and that fecal incontinence and defecation disorder occurred at rates of $0 \%-71 \%$ and $12 \%-74 \%$, respectively, in their long-term study. According to these studies, anterior resection syndrome may be a semipermanent change in the anorectal function rather than a temporary change after surgery [13]. A retrospective study in which the effects of biofeedback therapy were investigated in 513 patients with nonsurgical fecal incontinence reported that the FI-QoL was improved in more than $70 \%$ of the patients, that the incidence of fecal incontinence decreased and that the maximum anal-sphincter-muscle pressure increased [14]. In addition, a long-term study on rectal irrigation reported a decrease in the frequency of fecal incontinence and an increase in the FI-QoL [15]. Furthermore, a study on the effects of sacral nerve stimulation reported that fecal incontinence decreased and defecation-delaying ability increased in both the normal sphincter muscle group and the decreased sphincter muscle group [16]. Mechanistically, sacral nerve stimulation directly stimulates the anal sphincter muscle and increases the resting and the squeezing pressures [17]. As of yet, anterior resection syndrome still has no definitive and effective therapy. These results suggest that antidiarrheal agents, anal plugs, biofeedback therapy, and sacral nerve stimulation have potential for ameliorating symptoms such as fecal incontinence, an urgent defecation desire, and defecation disorder.

Many reports on defecation function disorder have been published. Ho et al. [18] reported that in patients with an excessive number of defecations and with fecal incontinence after an anterior resection or total colectomy, biofeedback therapy decreased the number of defecations and the degree of fecal incontinence. Another group investigated the defecation function and the FIQoL according to the self-anal-sphincter-muscle exercise status of 22 patients who received a sphincter-saving low anterior resection and reported a FI-QoL improvement due to the self-anal-sphincter-muscle exercise [19]. Furthermore, Visser et al. [20], as a result of their systematic literature review, reported that pelvic-floormuscle-strengthening rehabilitation therapy after a low anterior resection contributed to improved rectal pressure, WIS, and FIQOL score.

Diversion colitis refers to inflammation due to the halting of fecal flow at a nonfunctional segment of the distal colon after an ileostomy or colostomy [21]. Its major symptoms are abdominal pain, tenesmus, mucous stool, and bloody discharge [22]. Therefore, patients with diversion colitis easily experience discomfort, anxiety, and FI-QoL degradation.

In the present study, we found no differences in the WISs and 
the FI-QoL scores between the BG and the CG. However, pelvicfloor-muscle-strengthening rehabilitation therapy, such as anal sphincter muscle exercise and/or biofeedback therapy, alleviated the symptoms of patients whose anorectal function had been diminished due to diarrhea or an urgent defecation desire after colorectal surgery. Therefore, conservative management is preferentially used for patients with diarrhea and fecal incontinence after ileostomy closure. Nevertheless, a more detailed study on the effectiveness of selective application of biofeedback therapy is needed for patients with symptoms of severe diarrhea and fecal incontinence. In conclusion, based on the observations that the maximum squeezing pressures, the WISs, and the FI-QoL scores increased regardless of biofeedback therapy, we terminated this study early because the benefits of biofeedback over conservative management were not being demonstrated.

\section{CONFLICT OF INTEREST}

No potential conflict of interest relevant to this article was reported.

\section{REFERENCES}

1. Shirley F, Kodner IJ, Fry RD. Loop ileostomy: techniques and indications. Dis Colon Rectum 1984;27:382-6.

2. Huser N, Michalski CW, Erkan M, Schuster T, Rosenberg R, Kleeff J, et al. Systematic review and meta-analysis of the role of defunctioning stoma in low rectal cancer surgery. Ann Surg 2008;248:5260.

3. Roe AM, Warren BF, Brodribb AJ, Brown C. Diversion colitis and involution of the defunctioned anorectum. Gut 1993;34:382-5.

4. Lacima G, Pera M, Amador A, Escaramis G, Pique JM. Long-term results of biofeedback treatment for faecal incontinence: a comparative study with untreated controls. Colorectal Dis 2010;12: 742-9.

5. Ozturk R, Niazi S, Stessman M, Rao SS. Long-term outcome and objective changes of anorectal function after biofeedback therapy for faecal incontinence. Aliment Pharmacol Ther 2004;20:667-74.

6. Norton C, Chelvanayagam S, Wilson-Barnett J, Redfern S, Kamm MA. Randomized controlled trial of biofeedback for fecal incontinence. Gastroenterology 2003;125:1320-9.

7. Norton C, Cody JD, Hosker G. Biofeedback and/or sphincter exercises for the treatment of faecal incontinence in adults. Cochrane Database Syst Rev 2006;(3):CD002111.

8. Oliveira L, Pfeifer J, Wexner SD. Physiological and clinical outcome of anterior sphincteroplasty. Br J Surg 1996;83:502-5.

9. Rockwood TH, Church JM, Fleshman JW, Kane RL, Mavrantonis
C, Thorson AG, et al. Fecal Incontinence Quality of Life Scale: quality of life instrument for patients with fecal incontinence. Dis Colon Rectum 2000;43:9-16.

10. Kim KH, Yu CS, Yoon YS, Yoon SN, Lim SB, Kim JC. Effectiveness of biofeedback therapy in the treatment of anterior resection syndrome after rectal cancer surgery. Dis Colon Rectum 2011;54: 1107-13.

11. Pedersen IK, Christiansen J, Hint K, Jensen P, Olsen J, Mortensen PE. Anorectal function after low anterior resection for carcinoma. Ann Surg 1986;204:133-5.

12. Bryant CL, Lunniss PJ, Knowles CH, Thaha MA, Chan CL. Anterior resection syndrome. Lancet Oncol 2012;13:e403-8.

13. van Duijvendijk P, Slors JF, Taat CW, van Tets WF, van Tienhoven $\mathrm{G}$, Obertop H, et al. Prospective evaluation of anorectal function after total mesorectal excision for rectal carcinoma with or without preoperative radiotherapy. Am J Gastroenterol 2002;97:22829.

14. Byrne CM, Solomon MJ, Young JM, Rex J, Merlino CL. Biofeedback for fecal incontinence: short-term outcomes of 513 consecutive patients and predictors of successful treatment. Dis Colon Rectum 2007;50:417-27.

15. Koch SM, Rietveld MP, Govaert B, van Gemert WG, Baeten CG. Retrograde colonic irrigation for faecal incontinence after low anterior resection. Int J Colorectal Dis 2009;24:1019-22.

16. Tan E, Ngo NT, Darzi A, Shenouda M, Tekkis PP. Meta-analysis: sacral nerve stimulation versus conservative therapy in the treatment of faecal incontinence. Int J Colorectal Dis 2011;26:275-94.

17. Michelsen HB, Buntzen S, Krogh K, Laurberg S. Rectal volume tolerability and anal pressures in patients with fecal incontinence treated with sacral nerve stimulation. Dis Colon Rectum 2006;49: 1039-44.

18. Ho YH, Chiang JM, Tan M, Low JY. Biofeedback therapy for excessive stool frequency and incontinence following anterior resection or total colectomy. Dis Colon Rectum 1996;39:d1289-92.

19. Liu CH, Chen CH, Lee JC. Rehabilitation exercise on the quality of life in anal sphincter-preserving surgery. Hepatogastroenterology 2011;58:1461-5.

20. Visser WS, Te Riele WW, Boerma D, van Ramshorst B, van Westreenen HL. Pelvic floor rehabilitation to improve functional outcome after a low anterior resection: a systematic review. Ann Coloproctol 2014;30:109-14.

21. Son DN, Choi DJ, Woo SU, Kim J, Keom BR, Kim CH, et al. Relationship between diversion colitis and quality of life in rectal cancer. World J Gastroenterol 2013;19:542-9.

22. Whelan RL, Abramson D, Kim DS, Hashmi HF. Diversion colitis. A prospective study. Surg Endosc 1994;8:19-24. 